\title{
CINTILOGRAFIA ÓSSEA NO CÂNCER DE PRÓSTATA*
}

\author{
Benedita Andrade Leal de Abreu' ${ }^{1}$, Gerardo Aguiar Chaves², José Soares Junior ${ }^{3}$, \\ Evandro Leal de Abreu ${ }^{4}$, Emanuel Augusto de Carvalho Fontes ${ }^{5}$, Everardo Leal Abreu ${ }^{4}$, \\ José Wagner Bona ${ }^{5}$
}

\begin{abstract}
Resumo Este estudo propõe-se a revisar os aspectos de indicação da cintilografia óssea no estadiamento e acompanhamento dos pacientes com câncer de próstata. Os autores fazem um levantamento da literatura e analisam os critérios de indicação do exame, comparando-o com outras modalidades propedêuticas na avaliação desses pacientes. As aplicações da cintilografia óssea no câncer de próstata são estabelecidas segundo os novos parâmetros descritos na literatura, levando em conta, ainda, a relação custo/benefício.

Unitermos: Cintilografia óssea; Câncer de próstata.
\end{abstract}

Abstract Bone scintigraphy in patients with prostate cancer.

The aim of this study is to look over the indications for bone scintigraphy in the staging and follow-up of patients with prostate cancer. The authors reviewed the literature on the subject and analyzed the criteria used for indication of this test, and compared it with other methods for the evaluation of patients with this condition. Clinical applications of bone scintigraphy in prostate cancer are established according to new parameters described in the literature, also taking into consideration its cost-effectiveness.

Key words: Bone scintigraphy; Prostate cancer.

\section{INTRODUÇÃO}

A cintilografia óssea utilizando compostos difosfonados marcados com $\mathrm{Tc}^{99 \mathrm{~m}}$ é o método mais usado na detecção e seguimento das metástases do esqueleto ${ }^{(\mathbf{1})}$. Áreas de concentração aumentada do radiotraçador na cintilografia óssea são consideradas metástases; se existirem dúvidas acerca desta concentração anormal do radiofármaco, exames radiológicos complementares são realizados para afastar doenças benignas ${ }^{(2)}$. Com o surgimento de novas técnicas anatômicas como a ressonância magnética (RM) e a tomografia computadorizada (TC), houve um aumento potencial de formas de avaliação $^{(3,4)}$, entre-

* Trabalho realizado no Serviço de Medicina Nuclear do Centro Bionuclear de Diagnóstico, no Serviço de Urologia Oncológica do Hospital São Marcos e no Serviço de Urologia da Universidade Federal do Piauí (UFPI), Teresina, PI.

1. Médica Nuclear, Membro Titular do Colégio Brasileiro de Radiologia e Diagnóstico por Imagem (CBR), Doutoranda de Radiologia da Faculdade de Medicina da Universidade de São Paulo (FMUSP).

2. Professor Titular, Chefe da Clínica Urológica do Hospital Getúlio Vargas - UFPI.

3. Médico Chefe do Serviço de Medicina Nuclear e Imagem Molecular do Instituto do Coração do Hospital das Clínicas da FMUSP.

4. Estudantes de Medicina da UFPI.

5. Médicos Urologistas do Serviço de Urologia Oncológica do Hospital São Marcos.

Endereço para correspondência: Dra. Benedita A. Leal de Abreu. Rua Desembargador Pires de Castro, 489, Sul. Teresina, PI, 64001-390. E-mail: bionuclear@uol.com.br

Recebido para publicação em 30/7/2004. Aceito, após revisão, em 6/8/2004. tanto, a cintilografia óssea manteve-se com a vantagem de ser um método funcional e uma técnica de imagem inerentemente sensível ${ }^{(5)}$. Com o advento do antígeno prostático específico (PSA), surgiram novas abordagens e grandes discussões sobre o papel e importância da cintilografia óssea no estadiamento e acompanhamento dos pacientes com câncer de próstata ${ }^{(6-15)}$.

Neste artigo foram revisados artigos da literatura no período de 1976 até 2003, para se chegar ao papel atual da cintilografia óssea na avaliação de tais pacientes e sua correlação com o PSA, seja no seu estadiamento, no seguimento sistemático ou no estudo de pacientes sintomáticos no seu curso evolutivo.

\section{DISCUSSÃO}

\section{Câncer de próstata}

O câncer de próstata representa crescente problema de saúde pública nas sociedades ocidentais, nas quais a longevidade tem aumentado progressivamente. É caracteristicamente uma doença do homem idoso, e com o aumento da sobrevida humana, a importância do estudo e do tratamento do câncer de próstata tem crescido. No ano de 2002, nos Estados Unidos, estima-se que foram diagnosticados 189.000 novos casos e mais de 30.000 homens morreram de câncer de próstata ${ }^{(16)}$.
Para determinar a história natural do câncer de próstata, seus parâmetros clínicos e patológicos devem ser definidos. O câncer de próstata é um tumor de crescimento lento, com progressão não linear ${ }^{(2)}$. O local mais comum de disseminação hematogênica do câncer de próstata é o osso $^{(2)}$. A presença ou não de metástase óssea à época do diagnóstico é dado fundamental que direciona o tratamento ${ }^{(1)}$.

Embora atualmente existam muitas controvérsias a respeito da detecção e do manuseio, tanto da doença localizada como da metastática ${ }^{(5)}$, o estadiamento clínico é importante para garantir que o paciente tenha a modalidade terapêutica mais adequada para o seu caso ${ }^{(1)}$. O aspecto mais importante do estadiamento é a detecção de metástases e os métodos não invasivos são de grande importância na detecção dessas lesões ${ }^{(1)}$.

A maioria das morbidades e mortalidade no câncer de próstata avançado devese direta ou indiretamente ao comprometimento ósseo metastático, incluindo dor óssea, fraturas, imobilidade ${ }^{(16)}$. Na década passada, nos Estados Unidos (1994), 50\% a $60 \%$ dos pacientes com câncer de próstata tinham metástases ósseas ao serem diagnosticados ${ }^{(5)}$. A disseminação dos programas para detecção precoce e a introdução do teste de PSA têm aumentado a taxa de diagnóstico em fases mais iniciais ${ }^{(3,5)}$. 
Metástases ósseas são sabidamente um fator de mau prognóstico. Dos pacientes com câncer de próstata que desenvolvem metástase, 50\% morrem dentro de 30 me$\operatorname{ses}^{(2)}$. Na autópsia dos pacientes que morreram de câncer avançado de próstata, as metástases ósseas estavam presentes em $80 \%$ a $90 \%$ dos $\operatorname{casos}^{(\mathbf{1 6})}$.

\section{A cintilografia óssea}

A captação dos difosfonatos pelo osso na imagem cintilográfica depende tanto do fluxo sanguíneo local como da atividade osteoblástica. Embora o mecanismo de captação não seja ainda completamente compreendido, os difosfonatos são provavelmente incorporados nos cristais de hidroxiapatita na superfície óssea ${ }^{(\mathbf{1 7})}$. O agente mais largamente utilizado como traçador ósseo é ainda o MDP-Tc ${ }^{99 m}$, oferecendo ótimo contraste entre o tecido normal e doente, embora existam outros difosfonatos como alternativa para a realização da cintilografia óssea $^{(\mathbf{1 7})}$.

A cintilografia óssea, pela possibilidade de confirmação visual que proporciona, é o método de imagem mais apropriado para detectar metástases múltiplas no esqueleto (Figuras 1, 2 e 3). Além da vantagem de visibilizar, ao mesmo tempo, as metástases de todo o esqueleto em um só estudo, identifica as lesões que causam sintomas e também avalia áreas com risco potencial de fraturas ${ }^{(17)}$.

Antes do uso disseminado do PSA, vários estudos compararam a cintilografia óssea no câncer de próstata com outras formas de investigação, como avaliação clínica, raios-X simples, fosfatase alcalina e fosfatase ácida, sendo que a cintilografia ósea sempre se mostrou superior a qualquer desses métodos ${ }^{(7-10)}$.

Nos estudos de Schaffer e Pendergrass ${ }^{(10)}$ demonstrou-se que dos pacientes com cintilografia óssea positiva $43 \%$ não tinham dor óssea significativa, 39\% apresentavam níveis de fosfatase ácida prostática normais e $23 \%$ mostravam valores normais de fosfatase alcalina, evidenciando que a cintilografia óssea é mais sensível que os níveis de fosfatase ácida, fosfatase alcalina, sintomas clínicos, raios- $X$ convencional ou qualquer das combinações destes para detectar metástases ósseas nos adenocarcinomas de próstata ${ }^{(10)}$.

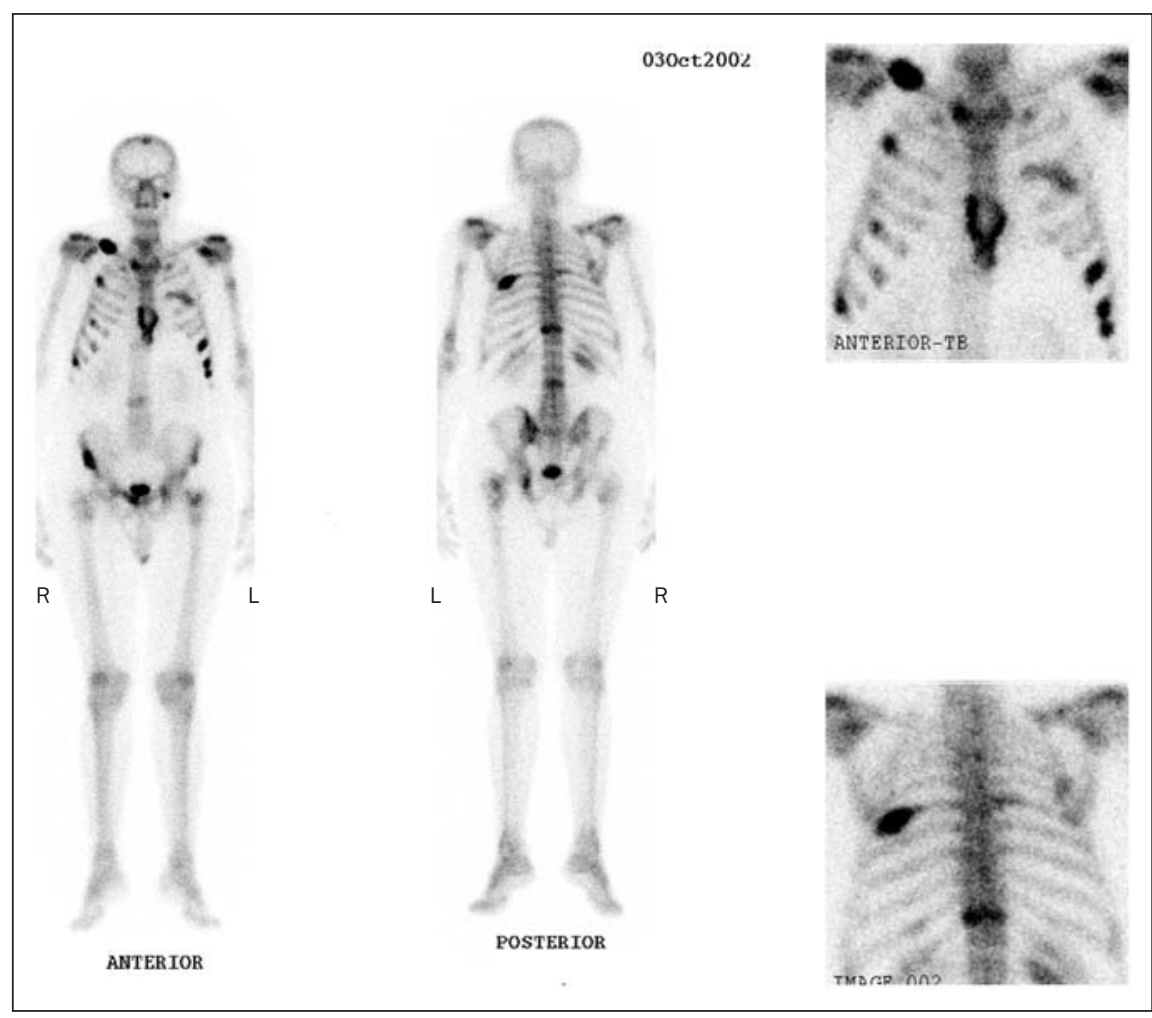

Figura 1. Paciente de 69 anos de idade com adenocarcinoma de próstata recentemente diagnosticado. Negava dor óssea. PSA = 8,1 $\mu \mathrm{g} /$ l. Cintilografia óssea: múltiplas lesões focais.

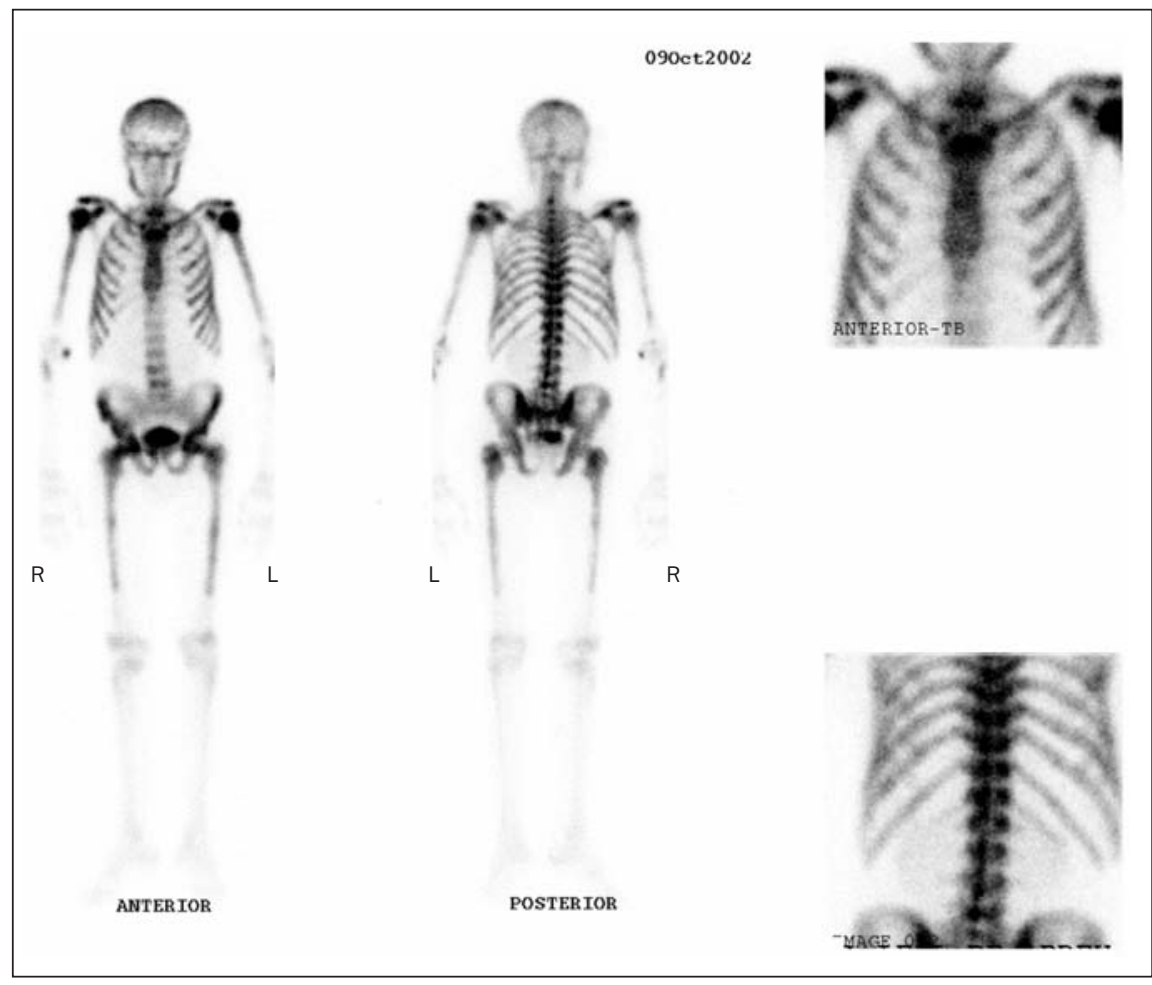

Figura 2. Paciente de 73 anos de idade com adenocarcinoma de próstata recentemente diagnosticado. Dores ósseas intensas. PSA $=250 \mu \mathrm{g} / \mathrm{l}$. Cintilografia óssea: ausência de imagens renais; metástases ósseas disseminadas. 


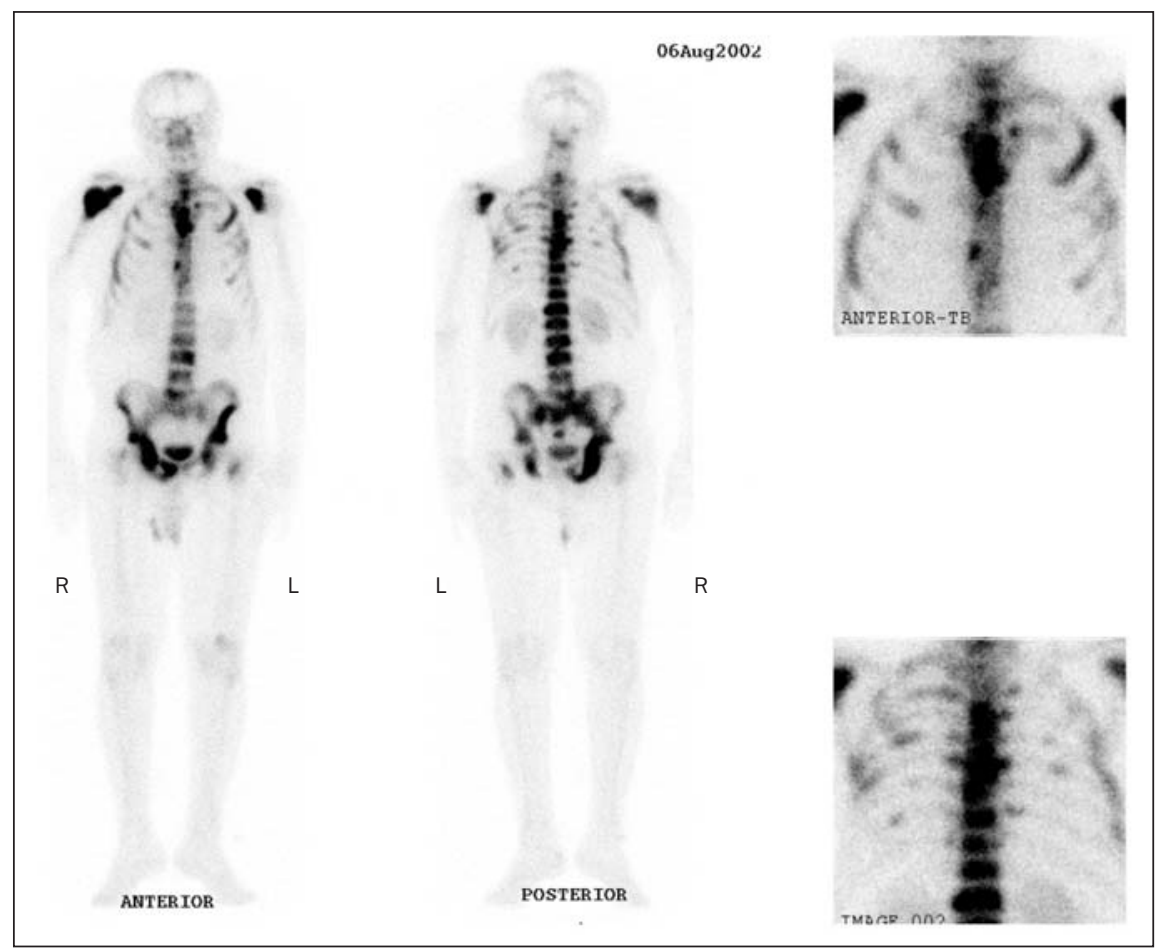

Figura 3. Paciente de 67 anos de idade. RX: artrose de coluna dorsal e lesões escleróticas difusas da bacia. PSA = $125 \mu \mathrm{g} /$ l. Adenocarcinoma de próstata. Cintilografia óssea: lesões metastáticas disseminadas.

Rana et al. ${ }^{(\mathbf{1 1})}$ classificaram os padrões de metástases ósseas e estudaram o seu significado prognóstico numa série consecutiva de 169 pacientes com câncer de próstata, dividindo-os em três grupos com significativas diferenças de sobrevida, apesar de receberem o mesmo tratamento hormonal. Este aspecto seria de utilidade para estudos comparativos de eficácia terapêutica, pois poderia estratificar os pacientes e ser um poderoso indicador prognóstico.

Rigaud et al. ${ }^{(2)}$ descreveram o valor da cintilografia óssea considerando a distribuição das metástases no esqueleto; eles estudaram 86 pacientes com câncer de próstata e metástases ósseas submetidos a terapia de deprivação androgênica como monoterapia e os classificaram de acordo com os locais de predominância das metástases na cintilografia óssea. Encontraram que pacientes com metástases axiais (coluna, pelve e crânio) têm melhor prognóstico que os com metástases apendiculares (arcos costais e membros) $^{(2)}$.

Wolff et $a .^{\left({ }^{(12)}\right.}$, num estudo retrospectivo de 359 pacientes, questionam se a cintilografia óssea pode ser omitida com segurança no estadiamento de pacientes com câncer de próstata, quando os níveis de PSA sérico são inferiores a $10 \mu \mathrm{g} / \mathrm{l}$, porque no seu estudo o valor preditivo negativo do PSA foi alto (96\%), mas o seu valor preditivo positivo foi de $50 \%$.

Wymenga et al. ${ }^{(\mathbf{1 3})}$, num estudo de 363 pacientes, encontraram cintilografia óssea positiva em 19 de 144 pacientes com níveis de PSA < $20 \mu \mathrm{g} / \mathrm{l}$, e destes, 14 tinham PSA $<10 \mu \mathrm{g} / \mathrm{l}$. Nesse estudo, os níveis de fosfatase alcalina se correlacionaram melhor com os achados de cintilografia óssea anormal que os níveis de $\operatorname{PSA}^{(\mathbf{1 3})}$. A maior incidência de metástases ósseas em pacientes com níveis de PSA mais baixos que nos estudos anteriores $^{(\mathbf{4 , 6 , 1 8 , 1 9 )}}$ deveu-se à maior proporção de pacientes com carcinoma pouco ou moderadamente diferenciado ${ }^{(\mathbf{1 3})}$.

Lee e Oesterling ${ }^{(20)}$, num estudo de 631 pacientes, encontraram que estadiamento clínico, escore de Gleason e PSA são todos preditores independentes de cintilografia óssea positiva em pacientes recentemente diagnosticados de câncer de prósreduzidos excluindo-se a realização da cintilografia óssea em pacientes de baixo risco (T2b ou menor, Gleason de 2-7 e PSA tata, e que, portanto, os gastos podem ser baixo), sendo que a cintilografia deve ser considerada em todos os outros casos. Se o paciente tem dor óssea, a cintilografia deve ser realizada independentemente dos níveis de $\operatorname{PSA}^{(\mathbf{2 0 )}}$

No que diz respeito ao seguimento de pacientes com câncer de próstata que foram submetidos a prostatectomia, a cintilografia óssea seria parte rotineira do acompanhamento sistemático, no entanto, com o advento do PSA, novas propostas têm sido sugeridas para a monitoração dos pacientes no pós-operatório. Segundo Terris et al. ${ }^{(21)}$, a cintilografia óssea é de grande contribuição quando no curso do seguimento os níveis de PSA tornam-se detectáveis ou o paciente desenvolve sintomas de dor óssea.

Caglar e Tuncel ${ }^{(\mathbf{1 4})}$ exemplificam casos em que o PSA é de pouco valor para predizer metástases ósseas: a) quando o paciente está em uso de terapia hormonal, pois a expressão do PSA é diretamente influenciada pelo status androgênico; b) nos casos de câncer pouco diferenciado. No curso da terapia hormonal ${ }^{(\mathbf{1 5})}$, pacientes com níveis normais ou baixos de PSA podem ter evidência de progressão metastática. Nestes casos, a cintilografia óssea é fundamental na detecção das metástases.

O papel atual da cintilografia óssea na avaliação dos pacientes com câncer de próstata, tanto no seu estadiamento quanto no seguimento, seja de pacientes sintomáticos como assintomáticos, tem sido tema de muita discussão e controvérsia. Muitos dos estudos, principalmente americanos, enfocam a relação custo/benefício. Vale lembrar que o custo médio de uma cintilografia óssea nos Estados Unidos é de

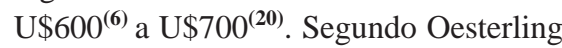
et al. ${ }^{(6)}$, em pacientes recentemente diagnosticados de câncer de próstata com concentração sérica de PSA de $10 \mu \mathrm{g} / 1$ ou menos e sem sintomas de dor óssea, a cintilografia óssea não parece ser necessária, podendo isto significar grande economia para os sistemas de saúde.

O estudo de Terris et al. ${ }^{(\mathbf{2 1})}$ avalia a combinação da cintilografia óssea com o PSA no acompanhamento de pacientes submetidos a prostatectomia radical e que à época da cirurgia não tinham evidência de doença óssea metastática. Esses autores voltam a valorizar a cintilografia como 
importante parte da investigação pré-operatória no estadiamento, para futuras comparações no seguimento desses pacientes ${ }^{(\mathbf{2 1})}$. Ressaltam que se o paciente se queixa de dor óssea, apesar de níveis indetectáveis de PSA, deve ser submetido à cintilografia óssea para diagnóstico, porque outro problema em potencial é a produção de auto-anticorpos PSA, que podem mascarar a detecção do PSA sérico ${ }^{(\mathbf{2 1})}$.

\section{Antígeno prostático específico}

O PSA é produzido por células acinares e epiteliais ductais de tecido prostático normal, hiperplásico e carcinomatoso. Foi primeiramente isolado em $1979^{(3)}$. No início dos anos 90 ganhou popularidade como o marcador tumoral mais importante no manuseio do câncer de próstata.

Os níveis séricos do PSA dependem do tamanho do tumor, do seu grau de diferenciação e do estadiamento ${ }^{(3)}$. Um estudo multicêntrico de 4.133 homens mostrou uma relação linear entre os níveis aumentados de PSA e a presença de doença extraprostática: $64 \%$ dos que apresentavam níveis < $4 \mu \mathrm{g} / \mathrm{l}$ e somente $9 \%$ dos que apresentavam PSA sérico > $50 \mu \mathrm{g} / \mathrm{l}$ tinham doença confinada ao órgão(3).

$\mathrm{O}$ advento do PSA sérico e a implementação dos programas de "screening" para detecção precoce do câncer de próstata têm determinado mudanças no estágio patológico em que o diagnóstico é realizado. Mais pacientes têm sido diagnosticados com tumores de menor volume e tem diminuído dramaticamente o índice de doença metastática ${ }^{(2)}$.

Vários estudos correlacionaram os níveis de PSA com a probabilidade de positividade da cintilografia óssea, tanto no estadiamento como no seguimento dos pacientes com câncer de próstata ${ }^{(\mathbf{1}, 6,13-15,18-30)}$. Chybowski et al. ${ }^{(\mathbf{1 8})}$, num estudo randomizado de 521 pacientes, avaliaram a capacidade do PSA de predizer os resultados da cintilografia óssea em pacientes recentemente diagnosticados de câncer de próstata, relatando que nível de PSA menor que $20 \mu \mathrm{g} / \mathrm{l}$ tem altíssimo valor preditivo negativo $(97,7 \%)$ para achados de cintilografia óssea, e que nenhuma das outras variáveis avaliadas, como estadiamento clínico, grau tumoral (Mayo "grade"), fosfatase ácida e fosfatase alcalina prostática ou combinação destas, foi de maior poder preditivo que o PSA isoladamente.

Por outro lado, níveis baixos ou indetectáveis de PSA podem ser encontrados em pacientes com doença metastática progressiva, na dependência do status androgênico do paciente, do desenvolvimento de auto-anticorpos, do grau de indiferencia-

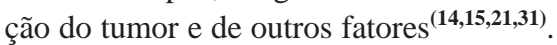

Outro dado importante é que concentrações aumentadas de PSA são também achadas em inflamações agudas e crônicas e em hipertrofias prostáticas benignas ${ }^{(32)}$. A densidade do PSA (que leva em consideração o volume glandular) dá uma melhor discriminação entre doença benigna e maligna $^{(6)}$. A densidade do PSA da zona de transição melhora o diagnóstico em pacientes com níveis intermediários de $\mathrm{PSA}^{(\mathbf{3})}$.

\section{CONCLUSÃO}

O método mais largamente aceito para detectar disseminação tumoral no esqueleto é a cintilografia óssea, sendo ela geralmente realizada como procedimento de rotina em todo homem com câncer de próstata recentemente diagnosticado. Estudos atuais têm usado a combinação PSA sérico, escore de Gleason e estágio clínico para selecionar os pacientes que necessitariam de cintilografia óssea no estadiamento, isto levando em conta a economia para os sistemas de saúde de se excluir este procedimento nos pacientes com baixo risco de metástases óssea. Alguns autores, todavia, como Gerber e Chodak ${ }^{(\mathbf{2 2})}$, questionam se o benefício de reduzir os custos, omitindo a cintilografia óssea em um grupo selecionado de pacientes, compensaria a desvantagem de estadiar incorretamente mesmo um pequeno porcentual de casos.

Em linhas gerais, não se discute que a cintilografia óssea, comprovadamente, não pode ser omitida em pacientes com fatores de mau prognóstico, incluindo altos níveis de PSA, alto escore de Gleason e estadiamento clínico avançado, ou que tenham sintomas de dor óssea.

Observamos, porém, que permanece não bem definido na literatura revisada quais níveis de PSA seriam seguros para dispensar a cintilografia óssea em pacientes recentemente diagnosticados de câncer de próstata: para alguns autores, níveis de
PSA menores que $10 \mu \mathrm{g} / \mathrm{l}^{(\mathbf{6 , 1 9 , 2 8})}$; para outros, o nível de segurança seria abaixo de $20 \mu \mathrm{g} / \mathrm{l}^{(\mathbf{4 , 2 9})}$; e outros ainda citam como seguros níveis menores que $50 \mu \mathrm{g} / \mathrm{l}^{(\mathbf{1 7 )}}$.

A cintilografia óssea continua sendo o padrão ouro na detecção de metástases ósseas no câncer de próstata ${ }^{(\mathbf{2 0})}$. Encontramos ainda, ressaltado por autores como Terris et al. ${ }^{(\mathbf{2 1})}$, que apesar de estudos mostrarem que os níveis pré-operatórios de PSA são de relevante utilidade no estadiamento do câncer de próstata, a cintilografia óssea permanece ainda como parte importante da rotina de avaliação pré-operatória para qualquer comparação futura.

\section{REFERÊNCIAS}

1. Lee N, Fawaaz R, Olsson CA, et al. Which patients with newly diagnosed prostate cancer need a radionuclide bone scan? An analysis based on 631 patients. Int J Radiat Oncol Biol Phys 2000;48:14431446.

2. Rigaud J, Tiguert R, Le Normand L, et al. Prognostic value of bone scan in patients with metastatic prostate cancer treated initially with androgen deprivation therapy. J Urol 2002;168(4 Pt 1):14231426.

3. Wilkinson BA, Hamdy FC. State-of-the-art staging in prostate cancer. BJU Int 2001;87:423-430.

4. Levran Z, Gonzalez JA, Diokno AC, Jafri SZ, Steinert BW. Are pelvic computed tomography, bone scan and pelvic lymphadenectomy necessary in the staging of prostatic cancer? Br J Urol 1995; 75:778-781

5. Dearnaley DP. Cancer of the prostate. BMJ 1994; 308:780-784.

6. Oesterling JE, Martin SK, Bergstralh EJ, Lowe FC. The use of prostate-specific antigen in staging patients with newly diagnosed prostate cancer. JAMA 1993;269:57-60.

7. O'Donoghue EPN, Constable AR, Sherwood T, Stevenson JJ, Chisholm GD. Bone scanning and plasma phosphatases in carcinoma of the prostate. Br J Urol 1978;50:172-177.

8. McGregor B, Tulloch AG, Quinlan MF, Lovegrove F. The role of bone scanning in the assessment of prostatic carcinoma. Br J Urol 1978;50:178-181.

9. Merrick MV, Ding CL, Chisholm GD, Elton RA. Prognostic significance of alkaline and acid phosphatase and skeletal scintigraphy in carcinoma of the prostate. Br J Urol 1985;57:715-720.

10. Schaffer DL, Pendergrass HP. Comparison of enzyme, clinical, radiographic, and radionuclide methods of detecting bone metastases from carcinoma of the prostate. Radiology 1976;121:431434.

11. Rana A, Chisholm GD, Khan M, Sekharjit SS Merrick MV, Elton RA. Patterns of bone metastasis and their prognostic significance in patients with carcinoma of the prostate. Br J Urol 1993;72:933936.

12. Wolff JM, Zimny M, Borchers H, Wildberger J, Buell U, Jakse G. Is prostate-specific antigen a reliable marker of bone metastasis in patients with newly diagnosed cancer of the prostate? Eur Urol 1998;33:376-381. 
13. Wymenga LFA, Boomsma JHB, Groenier K, Piers DA, Mensink HJA. Routine bone scans in patients with prostate cancer related to serum prostate-specific antigen and alkaline phosphatase. BJU Int 2001;88:226-230

14. Caglar M, Tuncel M. Preserved value of bone scintigraphy for the detection of skeletal metastases in prostate cancer patients with low prostate-specific antigen levels: effect of hormonal therapy and poor histologic differentiation. Clin Nucl Med 2002;27: 532-533.

15. Oommen R, Geethanjali FS, Gopalakrishnan G, et al. Correlation of serum prostate specific antigen levels and bone scintigraphy in carcinoma prostate. Br J Radiol 1994;67:469-471.

16. Clark PE, Torti FM. Prostate cancer and bone metastases: medical treatment. Clin Orthop Relat Res 2003;(415 Suppl):S148-157.

17. O'Sullivan JM, Cook GJR. A review of the efficacy of bone scanning in prostate and breast cancer. Q J Nucl Med 2002;46:152-159.

18. Chybowski FM, Keller JJL, Bergstralh EJ, Oesterling JE. Predicting radionuclide bone scan findings in patients with newly diagnosed, untreated prostate cancer: prostate specific antigen is superior to all other clinical parameters. J Urol 1991;145:313318 .

19. Rudoni M, Antonini G, Favro M, et al. The clinical value of prostate-specific antigen and bone scin- tigraphy in the staging of patients with newly diagnosed, pathologically proven prostate cancer. Eur J Nucl Med 1995;22:207-211.

20. Lee CT, Oesterling JE. Using prostate-specific antigen to eliminate the staging radionuclide bone scan. Urol Clin North Am 1997;24:389-394.

21. Terris MK, Klonecke AS, McDougall IR, Stamey TA. Utilization of bone scans in conjunction with prostate-specific antigen levels in the surveillance for recurrence of adenocarcinoma after radical prostatectomy. J Nucl Med 1991;32:1713-1717.

22. Gerber G, Chodak GW. Assessment of value of routine bone scans in patients with newly diagnosed prostate cancer. Urology 1991;37:418-422.

23. Rana A, Karamanis K, Lucas MG, Chisholm GD. Identification of metastatic disease by T category, Gleason score and serum PSA level in patients with carcinoma of the prostate. Br J Urol 1992;69:277281.

24. Miller PD, Eardley I, Kirby RS. Prostate specific antigen and bone scan correlation in the staging and monitoring of patients with prostatic cancer. Br J Urol 1992; 70:295-298.

25. Jacobson AF. Association of prostate-specific antigen levels and patterns of benign and malignant uptake detected on bone scintigraphy in patients with newly diagnosed prostate carcinoma. Nucl Med Commun 2000;21:617-622.

26. Wood DP Jr, Banks ER, Humphreys S, McRoberts
JW, Rangnekar VM. Identification of bone marrow micrometastases in patients with prostate cancer. Cancer1994;74:2533-2540.

27. Soh S, Kattan MW, Berkman S, Wheeler TM, Scardino PT. Has there been a recent shift in the pathological features and prognosis of patient treated with radical prostatectomy? J Urol 1997; $157: 2212-2218$

28. Gleave ME, Coupland D, Drachenberg D, et al. Ability of serum prostate-specific antigen levels to predict normal bone scans in patients with newly diagnosed prostate cancer. Urology 1996;47:708712 .

29. Kemp PM, Maguire GA, Bird NJ. Which patients with prostatic carcinoma require a staging bone scan? Br J Urol 1997;79:611-614.

30. Rees MA, Resnick MI, Oesterling JE. Use of prostate-specific antigen, Gleason score, and digital rectal examination in staging patients with newly diagnosed prostate cancer. Urol Clin North Am 1997;24:379-388.

31. Goldrath DE, Messing EM. Prostate specific antigen: not detectable despite tumor progression after radical prostatectomy. J Urol 1989;142:10821084

32. Nadler RB, Humphrey PA, Smith DS, Catalona WJ Ratliff TL. Effect of inflammation and benign prostatic hyperplasia on elevated serum prostate specific antigen levels. J Urol 1995;154:407-413. 